\title{
Evaluating the validity of diagnostic methods for scoliotic posture of primary school children
}

\author{
Artem Moment ${ }^{1 *}$, Denis Semenov ${ }^{2}$, Victor Golubkov ${ }^{1}$, Olga Mushtukova ${ }^{1}$ and Marina Semyonova ${ }^{1}$ \\ ${ }^{1}$ Institute of Education and Social Studies, Pskov State University, 180007, Pskov, Russia \\ ${ }^{2}$ Velikiye Luki State Academy of Physical Culture and Sports, 182105, Velikiye Luki, Russia
}

\begin{abstract}
Primary school children are exposed to asymmetric loads that lead to an imbalance of postural muscles and a violation of the body relative symmetry. Mass screening examinations in schools for scoliotic posture and scoliosis are conducted primarily through the Adams test. It has low reproducibility and a high frequency of false negative results. In practice, various methods for diagnosing posture disorders are also used. Some methods are based on the evaluation of postural muscles static endurance, elasticity and proportionality of their functional capabilities. Others are based on a comprehensive assessment of the ability to maintain an upright posture rationally. The third ones are based on the analysis of the tonic vibration and H-reflex of skeletal muscles on both sides of the vertebral column. All the above mentioned methods definitely have diagnostic potential. Therefore it was decided to determine the validity of some common methods for diagnosing posture disorders based on the analysis of their mutual congruence. Based on the results of the experiment, a reliable correlation between the studied parameters was established. It in turn allows us to judge the congruence of the considered diagnostic methods and, accordingly, the high probability of their validity.
\end{abstract}

\section{Introduction}

Posture disorders in children and adolescents are widely known to society. At the same time it is an underestimated problem. A child's posture is a mirror that reflects not only physical, but also emotional, mental health and social wellbeing.

Primary school age is a sensitive period of coordination abilities education. It is the age for starting the formation of the need for systematic physical exercise [1]. Rationally organized physical activity in childhood has a key effect on the correct development of a child, contributes to the formation and consolidation of optimal motor patterns and is a predictor of activity in adulthood [2]. The children of primary school age and older are particularly susceptible to asymmetric loads [3].It is shown that during this period of life, children's posture becomes very sensitive to changes in environmental conditions, lack of motor activity and the impact of low-intensity static load on the musculoskeletal system during school classes, preparation for them and leisure time in a sitting position in front of a computer screen, TV or other electronic gadget [4]. Prolonged sitting has a negative effect on the activity of the lumbar muscles. It leads to a redistribution of load on the passive structures of the vertebral column and, thus, contributes to the aggravation of muscle imbalance. It is a risk factor for posture disorders [2] and the occurrence of pain in the lumbar spine [5].

Epidemiological studies show that in $60-80 \%$ of primary school children have, to some extent, violations of posture $[6,7]$. Chinese scientists based on the results of a large-scale screening study confirmed previously available data on the frequency of deviations manifestations in the state of posture in children and found that with the onset of puberty, there is an aggravation of such violations [8]. It was found that postural disorders in the sagittal plane are very often combined with scoliotic configuration of the spine [7].

Low efficiency of measures for timely diagnosis and correction of deviations in the state of posture leads to violations of cardiorespiratory function, deterioration of metabolism in the body, irreversible changes in the skeletal system.

The most widely used methods of scoliotic posture diagnosis are: visual examination from the back when leaning forward (Adams test), radiography, computer topography [9]. Moreover, it should be noted that an in-depth examination of a child's posture is prescribed only if the result in the Adams test is positive. However, this test has a low reproducibility and a high frequency of false negative results [10], thus it cannot be a valid method for diagnosing scoliotic posture. Along with the methods described above, various pedagogical tests are used to determine the state of the muscular system, and in particular the static endurance of postural muscles, mobility of the vertebral column, muscle elasticity and proportionality of the paired postural muscles functional capabilities [11, 12]. Experimental methods for diagnosing posture disorders in primary school children are also used, based on the analysis of the equilibrium function parameters using a stabilometric platform and appropriate software [13, 14].

\footnotetext{
'Corresponding author: ar.moment@yandex.ru
} 
Researchers constantly point to the need to improve the effectiveness of school scoliosis early diagnosis and other types of posture disorders in the early stages before the end of the period of active growth [14-16]. The revealed deviations in the motor sphere of the child, allows to correct and fix the optimal motor patterns and automatism in a timely manner, thereby forming protective mechanisms against possible negative phenomena accompanying the growth spurt in the pubertal period [3].

The aim of the study is to determine the validity of some common methods for diagnosing scoliotic posture in primary school children based on the analysis of their mutual congruence.

\section{Materials and Methods}

The study involved 32 primary school children $(n=32)$ aged $7-11$ years. The half of the sample $(n=16)$ had postural disorders diagnosed by a doctor in the frontal plane. The other children $(n=16)$ had no similar disorders. The parents and the legal representatives of children were familiarized with the testing procedure, the algorithm of the experiment as a whole and its goals. After that we received their written consent to the participation in the experiment.

\subsection{Content of the experiment}

To improve the effectiveness of monitoring and managing the process of training the pupils, as well as to obtain objective data on the establishment of muscle imbalance, a set of tests was worked out.

\subsubsection{Evaluation of postural muscles static endurance}

- Static endurance of the abdominal muscles - holding the torso deflected by 60 degrees relative to the floor from the sitting position with the legs bent, hands behind the head, legs fixed. Note: the back should be straight. Testing stops when signs of fatigue begin to appear or at the request of the participant.

- Static endurance of the back muscles (lumbar spine) - holding the bent torso at an angle of 30 degrees to the floor from the initial position lying on the stomach, hands behind the head, legs fixed. Note: testing stops when signs of fatigue begin to appear, or at the request of the participant.

- Static endurance of the gluteal muscles - holding the straight legs drawn back from the position of lying on the stomach with the hands behind the head. Note: the Torso is pressed to the floor. Testing stops when signs of fatigue begin to appear or at the request of the participant.

\subsubsection{Estimation of the balance function}

To determine the characteristic features of children with posture disorders vertical stability in the frontal plane, a computer stabiloanalyzer with biofeedback "Stabilan - 01" produced by JSC Special R\&D Laboratory "RITM" was used.

The "Romberg test" was used as the research protocol.Placing stops on the stabiloplatform was carried out according to the "European" type. The duration of tests with open (OE) and closed (CE) eyes was 20 seconds each. The following stabilometric indicators were analyzed:

- Speed of the pressure center (PC) movement (V, millimeters per second) - the average speed of movement of the CP per unit of time;

- Coefficient of abrupt change in the direction of movement (\%) - an indicator that reflects the percentage of PC vectors (of the total number) that deviated from the previous vector by more than 45 degrees;

- $\quad \mathrm{PC}$ deviation in the frontal plane $(\mathrm{Q}(\mathrm{x}), \mathrm{mm})$ - normalized amplitude of the pressure center in the frontal plane;

- Quality of the balance function (\%) - an integral indicator that determines the "rationality" of maintaining a vertical posture.

\subsubsection{Evaluation of muscle imbalance}

The method of evaluating motor stereotype and muscle imbalance is based on the approach proposed by A. P. Shklyarenko (2002). The person tested was asked to perform 6 motor tasks. In the all tasks, the experts determined the symmetry of the performed movement.The criteria for evaluating symmetry were: no or minimal noticeable deviation of the torso or legs from the visual midline, as well as rotation of the torso or pelvis (depending on the motor task). The information on each repetition of the expert's motor task was entered into a specially developed protocol. It indicates the direction of the moving segment and rotation deviation, if any. At the end, the ratio of asymmetric movements to their total number was calculated. For example, the value of movement asymmetry of $30 \%$ corresponds to 18 motor errors out of the total number of movements equal to 60 . As a result, the presence of movements asymmetry determines not only the presence of a muscle imbalance, but also its localization.

Tests to evaluate muscle imbalance:

- Lifting the torso from the lying position on the back, hands behind the head. Note: raise the torso to the vertical, legs straight. Rectus and oblique abdominal muscles are tested. 
- Extension of the torso from a lying position on the stomach, hands behind the head. Note: the extension is performed at the maximum available comfortable amplitude. The muscles that straighten the spine are tested.

- Pull the legs back from the lying position on the stomach, hands behind the head. Note: do not bend your legs at the knee joints, keep your legs closed. Gluteal and lumbar muscles are tested.

- Lifting the legs forward from the supine position with the hands behind the head. Note: keep your legs straight and closed, and do not lift your lower back off the surface. The muscles of the front surface of the hip and abdomen are tested.

- Tilt from a standing position, a gymnastic stick at the top with the hands drawn back. Note: narrow stand legs apart, keep your arms straight, grip wider than your shoulders. The muscles of the shoulder girdle are tested.

- Flexion-extension of the arms in the supine position. Note: if it was impossible to complete the task due to insufficient physical fitness, testing was performed in simplified conditions with placing hands on a lift. The muscle complex is tested.

\subsection{Statistical analysis}

The statistical processing, analysis and systematization of the obtained data was carried out using the software Microsoft Excel, StatSoft Statistica 10 and StabMed 2.07.The analysis of the studied parameters distribution normality was performed using the Shapiro-Wilk W test, the relationship between data groups was made by applying the R. Spearman correlation analysis method.

To interpret the strength of the correlation relationship, the following normative values were adopted: $r-<0.25-$ weak correlation; $r-0,25-0,75$ - moderate correlation; $r->0.75$-strong correlation. When evaluating the reliability of the data obtained, a significance level of $5 \%$ was established.

\subsection{Limitations of the Research}

At the level of sample formation: the level of ordinary physical activity of children was not taken into account; the sample size was relatively small. At the level of statistical analysis: due to the small sample size, it was not possible to use strong parametric methods of data analysis. At the methodological level: the method of evaluating muscle imbalance requires objective refinement in relation to determining the significance level of the deviation and / or rotation of the moving body segment for its inclusion in the statistics of the imbalance.

The research authors are well aware of the difference between scoliotic posture and scoliotic disease. In this paper, we are talking about violations of posture and the initial stages of the vertebral column curvature without torsion of the vertebrae. In Russian literature, such phenomena are often called: "school scoliosis", "scoliosis", "violations of posture in the frontal plane", "scoliotic posture" and "scoliotic posture abnormality".

\section{Results and Discussion}

In order to confirm the validity of test tasks that characterize the imbalance of postural muscles in the presence of scoliotic posture, the level of relationship between the studied indicators was assessed by means of the correlation analysis.

The analysis of correlations between the studied indicators of static endurance, balance function and asymmetry of basic movements showed a fairly close relationship within the groups of tested abilities and between them.

Table1. Correlation between indicators of balance function and motor abilities in primary school children.

\begin{tabular}{cccccccccc}
\hline Parameters & 1 & 2 & 3 & 4 & 5 & 6 & 7 & 8 \\
\hline 1 & 1 & 0,68 & 0,49 & $-0,76$ & $-0,77$ & $-0,73$ & 0,78 & $-0,69$ \\
2 & 0,68 & 1 & 0,66 & $-0,81$ & $-0,84$ & $-0,80$ & 0,88 & $-0,82$ \\
3 & 0,49 & 0,66 & 1 & $-0,78$ & $-0,80$ & $-0,76$ & 0,74 & $-0,76$ \\
4 & $-0,76$ & $-0,81$ & $-0,78$ & 1 & 0,93 & 0,88 & $-0,88$ & 0,91 \\
5 & $-0,77$ & $-0,83$ & $-0,79$ & 0,93 & 1 & 0,87 & $-0,88$ & 0,89 \\
6 & $-0,73$ & $-0,80$ & $-0,80$ & $-0,76$ & 0,87 & 1 & $-0,84$ & 0,93 \\
7 & 0,78 & 0,87 & 0,74 & $-0,88$ & $-0,88$ & $-0,84$ & 1 & $-0,85$ \\
8 & $-0,69$ & $-0,82$ & $-0,76$ & 0,91 & 0,89 & 0,93 & $-0,85$ & 1 \\
\hline
\end{tabular}

Note: 1 - the static endurance of the abdominal muscles; 2 - the static endurance of the back muscles; 3 - static endurance of the gluteal muscles; 4 - speed of movement of the CP; 5 -coefficient of abrupt change in the direction of movement; 6-CP deviation in the frontal plane; 7 - quality of the balance function; 8 -asymmetry in the movements.

The indicators of static endurance of the muscles of the back protractors and pelvic retainers $(0.66)$ and the abdomen (0.68) have a moderate direct dependence.

It is worth noting that the correlation between the values of static endurance of the pelvic retainers and abdominal muscles is weaker $(0.49)$. The indicators of the balance function are strongly correlated with each other module $(0.73-$ 0.85), because they reflect the interdependent characteristics of the movement of the PC and the imbalance of the 
vertical posture. The value of the movement asymmetry is inversely related to the static endurance of the tested muscle groups. Moreover, a greater level of correlation is observed between the indicators of movements asymmetry and static endurance of the back protractor muscles $(-0.82)$, then the pelvic retainers $(-0.76)$ and to a lesser extent with the abdominal muscles (-0.69).

Concerning the relationship of various muscle groups static endurance to indicators of balance function we can also observe a correlation from a moderate to a strong level. However, as in the case of the correlation analysis between static endurance and asymmetry of basic movements, a higher level of correlation between the studied indicators of the balance function is observed with the indicators of the back protractor muscles static endurance and quality of the balance function (0.88), deviation of the pressure CP in the frontal plane (-0.80), coefficient of abrupt change in the direction of movement (-0.84), and the speed of the CP movement (-0.81). The correlation of abdominal muscles static endurance and quality of the balance function (0.78), deviation of the $\mathrm{PC}$ in the frontal plane $(-0.73)$, coefficient of abrupt change in the direction of movement (-0.77), speed of the PC movement (-0.76). The indices of static endurance of pelvic retainers muscles are in moderate correlations with quality of the balance function $(0.74)$, a strong inverse correlation with PC deviation in the frontal plane (-0.76), coefficient of abrupt change in the direction of movement ($0.80)$ and speed of the PC movement (-0.78).

The asymmetry of the basic movements and all the studied indicators of the balance function are strongly correlated. So, in inverse proportion to the magnitude of basic movements asymmetry measure is an indicator of the balance function quality $(-0.85)$, in direct proportion: PC deviation in the frontal plane (0.93), coefficient of abrupt change in the direction of movement (0.89), speed of the PC movement.

According to the results of the research by several independent groups of scientists a direct correlation was experimentally established between the indicators of isometric strength of the trunk and lower extremities muscles with the indicators of dynamic postural stability in primary school children [17-19].The direct dependence between the violations of posture in the frontal plane, the value of scoliotic deformation and asymmetry of movements was confirmed [20].Earlier researches experimentally confirmed the assumption of the posture disorders influence in the frontal plane on the ability of children to maintain a vertical posture rationally[13, 14,21].By registering the tonic vibration and H-reflex of skeletal muscles on both sides of the vertebral column in children with scoliotic posture, an imbalance of excitability of nerve centers innervating symmetrical muscle groups was revealed [22].

The muscular imbalance that we believe underlies such disorders contributes to the destabilization of motor automatisms and patterns. For example, it is noted that children in preschool and primary school age often have contracture of the lower extremities muscles, responsible for the symmetrical position of the pelvis and, as a result, the vertebral column [3]. The effect of altered afferent stimuli causes adaptive neurophysiological responses that underlie motor automatism and patterns [23]. As a result of neuroplastic processes, these changes are fixed at the level of cortical motor centers, forming a suboptimal motor stereotype.

\section{Conclusions}

Based on the results of the experiment, a reliable correlation between the parameters under study was established. It in turn allows us to judge the congruence of the diagnostic methods under consideration and, accordingly, the high probability of their validity.

Thus, the evaluation of the indicators that characterize the symmetry of movements, static endurance of postural muscles and the quality of postural function allows us to diagnose with a high degree of probability both existing and developing muscle imbalance in primary school children.

\section{References}

1. W. Chen, A. Hammond-Bennett, A. Hypnar, S. Mason, BMC Public Health, 18(1), 195 (2018)

2. A. Brzęk, A. Knapik, J. Sołtys, W. Gallert-Kopyto, A. Famuła-Wąż, R. Plinta, Medicine (Baltimore), 98(7), e14449 (2019)

3. A. Brzek, R. Plinta, Medicine (Baltimore), 95(12), e2855 (2016)

4. K. Maciałczyk-Paprocka, B. Stawińska-Witoszyńska, T. Kotwicki, et al. Eur J Pediatr. 176(5), 563-572 (2017)

5. F. Mörl, I. Bradl, J Electromyogr Kinesiol, 23(2), 362-368 (2013)

6. M.O. Kuzmina, Healthcare, education and safety, 3 (11), 68-75 (2017) (In Russ.)

7. E.I. Aukhadeyev, O.B. Sergeyeva, Physical education in prevention, treatment, rehabilitation: scientific-practical magazine, 3-4(34-35), 92-95 (2010) (In Russ.)

8. L. Yang, X. Lu, B. Yan, Y. Huang, iScience, 23(5), 101043 (2020)

9. R.M. Aroeira, E.B. de Las Casas, A.E. Pertence, M. Greco, J.M. Tavares, J Body w Mov Ther, 20(4), 832-843 (2016)

10. T. Karachalios, J. Sofianos, N. Roidis, G. Sapkas, D. Korres, K. Nikolopoulos, Spine (Phila Pa 1976), 24(22), 23182324 (1999).

11. A.P. Shklyarenko, D.A. Ulyanov, T.G. Kovalenko, Bulletin of Volgograd State University. Series 11: Natural Sciences, 22 - 27 (2016) (In Russ.)

12. A.S. Kuznetsov, Z.M. Kuznetsova, Russian Journal of Physical Education and Sport, 14(4), 5-7 (2019) 
13. V.V. Koss, Early diagnostics and prevention of progression of postural disorders and scoliosis of I-II degree among children in educational institutions: diss.abstract...Cand.Med.Sc. (2009) (In Russ.)

14. A.V. Moment, D.V. Semenov, Physical education and sport training: scientific-theoretical magazine 4(26), 96-102 (2018) (In Russ.)

15. F. Altaf, J. Drinkwater, K. Phan, A.K. Cree, Spine Deform, 5(5), 303-309 (2017)

16. R. Ningthoujam, Int J Phys Educ Health Sports Sci, (3), 2-8 (2014)

17. A.I. Ibrahim, Q.I. Muaidi, M.S. Abdelsalam, Z.M. Hawamdeh, A.A. Alhusaini, J Manipulative Physiol Ther. 36(9), 633-643 (2013)

18. B. Hirunyaphinun, S. Taweetanalarp, A. Tantisuwat, Hong Kong Physiother J. 39(2), 143-150 (2019)

19. U.H. Mitchell, A.W. Johnson, B. Adamson, J Strength Cond Res, 29(5), 1172-1179 (2015)

20. A.P. Shklyarenko, Physiological substantiation of Physical Education for scoliotic disease among children and teenagers: Diss....PhD in Biol. (2002) (In Russ.)

21. A.V. Moment, D.V. Semenov, Scientific notes of the University named after P.F. Lesgaft, 9 (139) 106 - 112 (2016) (In Russ.)

22. O.A. Polikarpova, Prevention of postural disorders by means of gymnastics among junior pupils based on individual approach: Diss....Cand.Ped. (2007) (In Russ.)

23. R. Pelletier, J. Higgins, D. Bourbonnais, BMC Musculoskelet Disord, 16, 25 (2015) 